\title{
A Note on Translation
}

Since the following work is essentially one of commentary on and interpretation of a body of theoretical thought, translation emerges, in this context, only as a special, limiting case of the same critical operation. The goal of all these interrelated activities is, of course, truth of statement and accuracy of comment, but, as in all interpretation, a certain "attitude" or "turn" is unavoidable. Throughout my study I have sought to make that interpretive point of view consistent and coherent, be it in narrative commentary or direct citation. For this reason I offer my own translations, except for one or two instances where the available versions seemed unimpeachable, in the quoted passages, regardless of whether the originals are in Czech, Russian, German or French. Even though I have tried to provide translations which are as exact and literal as possible, I have struggled not to lose sight of the demands of English idiomatic usage, and, in the battle between the two imperatives, I have usually opted for the latter. In short, unless otherwise indicated, all translations-and all responsibility-are mine. 